\title{
Optimal Weight Power System Design and Synthesis for More Electric Aircraft
}

\author{
Angel A. Recalde, Serhiy Bozhko,2 \\ School of Electrical and Electronic Engineering, University of Nottingham, NG7 2TU, UK \\ Escuela Superior Politecnica del Litoral ESPOL, EC 090112, Ecuador \\ Jason Atkin 3 \\ School of Computer Science, University of Nottingham, NG8 1BB, United Kingdom
}

\begin{abstract}
The synthesis of power distribution architecture for More Electric Aircraft requires optimization of weight in order to reduce energy consumption. Optimal weight of the power distribution system of the aircraft mainly depends on compliance of stringent safety requirements, as well as component selection and location. Safety is dictated by the reliability constraints that ensures distribution adequacy to maintain critical loads powered at all times. Optimal component selection and location is a combinatorial problem that determines a set of interconnected devices located in specific positions such that the resultant topology has minimum weight. Given the complexity in producing and solving such optimization formulation, this paper realizes a linear optimization design that is subject to reliability constraints to comply with safety requirements, and component selection and location constraints such that the weight of the electrical distribution system is minimized. The design formulation is presented as a Mixed Integer Linear Programming problem and a case study is used to exemplify the synthesis of a power distribution architecture that is optimal.
\end{abstract}

\section{Nomenclature}

Sets

$=$ set of available generators

$=$ set of available distribution paths (generator - load connections)

$=$ set of available distribution components

$=$ set of available connections between distribution components

$=$ set of selected distribution paths (generator - load connections), $K \subseteq \mathcal{E}$

Generator Selection and Generator-Load Pairing

$\mathcal{G}=(g, \mathcal{E}) \quad=\quad$ graph containing set of generators $g$ and distribution paths $\mathcal{E}$

$w_{s}, P_{s}^{G} \quad=$ weight, and power rating for generator $s$

$L_{l}, r_{\mathrm{TARGET}, l}=$ load demand and reliability target for load $l$

$g_{s} \quad=$ Boolean selection for generator $s$

$y_{s l} \quad=$ Boolean selection for distribution path that connects generator $s$ with load $l$

$r_{s l} \quad=$ reliability of distribution system or path that connects generator $s$ with load $l$

$u_{s h}, w_{h} \quad=$ weight linearization Boolean variable and coefficient

Power Distribution Design

$\mathcal{G}=(\mathcal{N}, \mathcal{A}) \quad=\quad$ graph containing set of components $\mathcal{N}$ and connections $\mathcal{A}$

1 Early Stage Researcher, angel.recalde@ nottingham.ac.uk

2 Institute for Aerospace Technology, serhiy.bozhko@nottingham.ac.uk

3 Faculty of Science, jason.atkin@nottingham.ac.uk 


$\begin{array}{ll}k, r_{s l} & =\text { distribution path }(k \in K, k \in \mathcal{E}), \text { reliability target for distribution path } k \\ P_{i j, k}, P_{i, k} & =\text { power flow on connection } i, j \text { and component } i \text { for distribution path } k \\ w_{i j}, w_{i} & =\text { fixed weight for connection } i, j ; \text { fixed weight for component } i \\ w_{i j}^{\mathrm{kW}}, w_{i}^{\mathrm{kW}} & =\text { variable weight for connection } i, j ; \text { variable weight for component } i \\ x_{i j}, x_{i} & =\text { Boolean selection for connection } i, j \text { and component } i \\ r_{i j}, r_{i} & =\text { reliability of connection } i, j, \text { reliability of component } i \\ d_{k}^{L L A D}, d_{k}^{G E N} & =\text { load demand and power generation of the distribution path } k \\ z_{i j, k}, z_{i, k} & =\text { Boolean variables to associate connection } i, j, \text { component } i \text { with distribution path } k \\ m_{i}, n_{i} & =\text { Abscissa and ordinate position of component } i \\ c_{o}^{\mathrm{AWG}} & =\text { Boolean variable to select a unique standard wire gauge if connection } i, j \text { is selected } \\ I_{o}^{\mathrm{AWG}}, r_{o}^{\mathrm{AWG}} & =\text { ampacity and resistance per-unit-of-length of standard gauge type } o \\ w_{o}^{\mathrm{AWG}} & =\text { weight per-unit-of-length of standard gauge type } o \\ q_{w} & =\text { Boolean variable to select alternative paths for distribution path } k \\ V_{p . u .}^{\mathrm{DROP}}, V_{i j}^{\mathrm{OP}} & =\text { permissible voltage drop (in p.u.), and operational voltage for connection } i, j\end{array}$

\section{Introduction}

The More Electric Aircraft (MEA) power distribution system consists of a set of interconnected components that performs power transmission and conversion to supply all loads with power at the required voltage level and format (AC or DC) [1], [2]. DC systems are very attractive in MEA due to the reduced number of conductors [3] and the inexistence of reactive power compared to AC systems [4]. In addition, among several wiring electrical configurations studied for MEA, DC systems proved to be the candidate that provides maximum power transfer and maximum weight to power ratio capability for the same voltage range [3]. Several DC distribution topologies have been proposed in MEA to provide adequate number of power conversion levels, high reliability, and high-power density [1], [5]-[7]. Hence, the main objective in determining a power system architecture for MEA is minimizing the amount of weight (reduce the system's payload) while exploiting safety and power transfer capabilities.

Safety specifications can be translated into a set of reliability constraints that ensures the power system comply with a certain reliability level (reliability metric) by assembling sufficient number of components and distribution paths [8], [9]. Several approaches based on reliability-based optimization problems [10] have included safety specifications as reliability constraints in linear optimization formulations which resolves for an optimum topology [11], [12]. These techniques can be applied either with iterative or sequential algorithms. In the former, the number of reliability constraints increase depending on the reliability requirements [11], so that the connectivity complexity is increased only if required by specification (otherwise, simpler topologies are feasible). In the latter, starting at an abstract representation (platform) of the system's functional and safety specifications, the design walks through a certain number of refinement steps until the platform is very close to final implementation [13]. Although these formulations synthesize a power system architecture based on functional and reliability requirements, there are further steps to be performed in order to bring the solution closer to a final implementation. This paper pretends to close this gap by exploring weight minimization via component selection and location while complying to a set of reliability constraints. The design exercise is formulated as a Mixed Integer Linear Programming problem (MILP) to experience the advantage of reaching optimality in polynomial time (depending on the number of variables) and using powerful MILP solvers available commercially. The rest of the paper is outlined as follows. Section III develops the design formulation to synthesize a power distribution architecture considering connectivity, reliability, component selection, and location constraints. Then, Section IV presents a linear transformation of the design problem. Section V exemplifies the use of the proposed formulation in the case study. Finally, Section VI comes to the conclusion of the paper.

\section{Design Formulation}

The power system design must comply with the functional and safety specifications through the enforcement of a set of connectivity and reliability constraints. In addition, constraints on the components' selection and location are included in an attempt to get closer to a final implementation where the MEA power distribution's weight is minimized.

The MEA power distribution system comprises a set of interconnected power sources and distribution devices that performs power generation, transmission, conversion, and distribution to supply power to loads. For the rest of the 
paper, the power sources are assumed to be generators driven by the aircraft' turbine engines. Given that the power conversion is driven by a power density $(\mathrm{kW} / \mathrm{kg})$ and the amount of power to convert, the weight of the power distribution system is independent from the generator-load combination. Hence, following a PBD methodology [14], the MEA power system is synthesized in two sequential steps [13], [15]: generator selection and generator-load pairing (GS\&GLP), and power distribution design (PDD). The former selects a number of power sources (generator selection) and determines which loads are supplied by those power sources at any time (generator-load pairs or distribution paths). Then, the latter synthesizes a power distribution topology whose number of components and their corresponding sizes depend on the previous generator-load pair arrangement or number of distribution paths [13], [16].

The design exercise requires to supply a pre-specified set of critical loads $L$ with functional and reliability requirements. Let a set of $g$ generators and a set of $\mathcal{E}$ distribution paths (generator-load connections) be represented by a template (graph) $\mathcal{G}=(g, \mathcal{E})$. Each generator $s$ has a set of parameters, i.e. weight $w_{s}$, reliability $r_{s}$, and power rating $P_{S}^{G}$. The GS\&GLP step attempts to select subsets $s \subseteq g$ and $K \subseteq \mathcal{E}$ of generators and distribution paths respectively that minimizes generation weight $w$, supply each of the critical loads $l$ (from the set $L$ ), and satisfies the connectivity and reliability constraints. Let Boolean $g_{s}$ determine the selection $\left(g_{s}=1\right)$ or rejection $\left(g_{s}=0\right)$ of a generator $s$ of the set $g$. The generation's weight minimization can be written as:

$$
\underset{w, g, y}{\operatorname{minimize}}\left(\sum_{s} w_{s} g_{s}\right)
$$

In (1), $w_{s}$ is the weight of generator $s$ and the product $w_{s} g_{s}$ equals $w_{s}$ if $g_{s}=1$ (otherwise, generator $s$ is not selected and its weight contribution is 0 ). The generator weight $w_{s}$ is assumed to be a function of the power rating $P_{s}^{G}$. The weight of the distribution system is not included in (1) because in the GS\&GLP step, the distribution system is represented as a set of parameter-less generator-load connections (distribution paths) whose number depends on the loads' reliability requirements. The power distribution parameters (which are considered independent from the generator-load arrangement) are optimized in the PDD step. The functional requirements are determined by a set of connectivity constraints and the reliability requirements enforces sufficient power generation capacity and distribution paths to supply critical loads.

The connectivity constraints allow each load $l$ to be connected to a generator $s$. Each of the selected generators $s$ must have a power rating $P_{S}^{G}$ greater than the minimum power rating $P_{\mathrm{MIN}}^{G}$ but less than the maximum power rating available $P_{\mathrm{MAX}}^{G}$. A set $H$ of $h$ commercial power rating values $P_{h}^{G}$ can be used to select a specific $P_{s}^{G}$ for generator $s$. Let a Boolean $u_{s h}$ select a power rating value $h \in H$ for generator $s$. Each generator has a unique power rating if selected, then,

$$
\sum_{h} u_{s h} \leq g_{s} \quad \forall s
$$

Let the Boolean $y_{s l}$ determine the selection $\left(y_{s l}=1\right)$ or rejection $\left(y_{s l}=0\right)$ of the distribution path $s l$ connecting generator $s$ to the load $l$. A load $l$ is connected to a generator $s$ if that generator has been selected, then,

$$
y_{s l} \leq \sum_{h} u_{s h} \quad \forall l, \forall s
$$

Each load is connected at least to one generator, then,

$$
\sum_{s} y_{s l} \geq 1
$$

The power rating $P_{h}^{G}$ of the generator $s$ is greater than or equal to the total load connected to it, then,

$$
\sum_{l} L_{l} y_{s l} \leq \sum_{h} P_{h}^{G} u_{s h} \quad \forall s
$$

For each load $l$, the reliability is considered as the availability of power supply on the load's terminal. Hence, there is a certain reliability target $r_{\text {TARGET, } l}$ for each load $l$ that needs to be achieved. Let $r_{s}$ be the reliability of the generator $s$, and $r_{s l}$ the reliability of each distribution path $y_{s l}$ (generator-load connection). When a load $l$ is connected to a generator $s$, the reliability on the load terminals is $r_{s l} r_{s}$, which is the reliability of a series-system. When a load $l$ is 
connected to more than one generator, the reliability of a parallel series-system can be applied. Then, the reliability of a load $l$ connected to multiples generators must be greater than or equal to $r_{\mathrm{TARGET}, l}$, then

$$
1-\prod_{s}\left(1-y_{s l} r_{s l} r_{s}\right) \geq r_{\mathrm{TARGET}, l} \quad \forall l
$$

The product $y_{s l} r_{s l}$ in (6) allows to set $r_{s l}$ to 0 if the distribution path $y_{s l}$ is not selected $\left(y_{s l}=0\right)$. As mentioned before, the outcome of the GS\&GLP step is a group of generators $s \subseteq g$ with their corresponding ratings $P_{h}^{G}$ and a group of distribution paths (generator-load connections) $K \subseteq \mathcal{E}$ with their corresponding reliabilities $r_{s l}$. The reliability constraint in (6) contains the distribution path's reliability variable $r_{s l}$ which is used as the reliability target of the distribution path in the PDD step.

The PDD step aims to synthesize a power distribution architecture that implements the distribution paths arrangement (generator-load connections) found in the GS\&GLP step. Consider a set $\mathcal{N}$ of power distribution components and a set $\mathcal{A}$ of feasible connections between the power distribution components. Let these two sets be represented by a template (graph) $\mathcal{G}=\{\mathcal{N}, \mathcal{A}\}$. Each component $i$ has a set of parameters, i.e. weight $w_{i}$, reliability $r_{i}$, and power conversion/transmission capacity $P_{i}$. Similarly, each connection $i, j$ has a set of parameters, i.e. weight $w_{i j}$, reliability $r_{i j}$, and a power flow $P_{i j}$. The PDD attempts to select a subset of components $n \subseteq \mathcal{N}$ and a subset of connections $a \subseteq \mathcal{A}$ that minimizes power distribution weight, while satisfying a group of connectivity and reliability constraints. The power distribution weight consists of a fixed weight (installation payload) and a variable weight that depends on the amount of power flow converted/transferred.

Let a Boolean $x_{i}$ select a component $i$ from the set $\mathcal{N}$ (all selected components will form the subset $n$ ). Also, let component $i$ have a fixed weight $w_{i}$, a variable weight $w_{i}^{\mathrm{KW}}$ that represents the ratio between a unit of weight and a unit of power converted/transferred, and a power flow $P_{i}$. Similarly, let a Boolean $x_{i j}$ select a connection (from the set $\mathcal{A}$ ) between component $i$ and $j$ (all selected connections will form the subset $a$ ). Let each connection $i, j$ have a fixed weight $w_{i j}$, a variable weight $w_{i j}^{\mathrm{KW}}$ that represents the ratio between a unit of weight and a unit of power transferred, and a power flow $P_{i j}$. The parameters $w_{i}^{\mathrm{KW}}$ and $w_{i j}^{\mathrm{KW}}$ (in $\mathrm{kg} / \mathrm{kW}$ ) are the inverse of the power density (in $\mathrm{kW} / \mathrm{kg}$ ) for components and connections respectively. Then, the total power distribution's weight (fixed weight plus variable weight) can be written in terms of selection variables $x_{i}, x_{i j}$ and power flows $P_{i}, P_{i j}$,

$$
\underset{P, x, v}{\operatorname{minimize}}\left[\left(\sum_{(i, j) \in \mathcal{A}} w_{i j} x_{i j}+\sum_{i \in \mathcal{N}} w_{i} x_{i}\right)+\left(\sum_{(i, j) \in \mathcal{A}} w_{i j}^{\mathrm{kW}} P_{i j}+\sum_{i \in \mathcal{N}} w_{i}^{\mathrm{kW}} P_{i}\right)\right]
$$

The first term in (7) is the summation of all fixed weights, and the second term is the summation of all variables weights (which depends on the amount of power flow converted/transferred). Given that the PDD step implements the solution of the GS\&GLP (constructing the selected distribution paths $y_{s l}$ ), there is a number of $K$ distribution paths that are built with selected components $x_{i}$ and connections $x_{i j}$. Each distribution path $k(k \in K, k$ exists if and only if $y_{s l}=1$ ) connects the generator $s$ and the load $l$ of its corresponding $y_{s l}$. It is possible that two or more distribution paths $k$ could share the same component $i$ and connection $i, j$ (depending on the reliability constraints also). Also, all the component's power flows can be expressed in terms of the connections' power flows $P_{i j}$. Let a connection $i, j$ have a power flow $P_{i j, k}$ for every distribution path $k$ such that $P_{i j}=\sum_{k \in K} P_{i j, k}$ (if connection $i, j$ serves only one distribution path $k$, then only one $P_{i j, k} \neq 0$, and all other $P_{i j, k}=0$ ), and let a component $i$ have a power flow $P_{i, k}$ for every distribution path $k$ such that $P_{i}=\sum_{k \in K} P_{i, k}$ (if component $i$ serves only one distribution path $k$, then only one $P_{i, k} \neq 0$, and all other $P_{i, k}=0$ ). Note that $P_{i, k}$ can be expressed in terms of the incoming (or outgoing) connections' power flows, i.e. $P_{i, k}=\sum_{j} P_{j i, k}$ such that the flow through component $i$ is the summation of all incoming (or outgoing) flows coming from other components $j$. As $P_{i, k}=\sum_{j} P_{j i, k}$, the component $i$ flow is $P_{i,}=\sum_{k \in K} \sum_{j} P_{j i, k}$. For any component $i$, it is assumed that summation of all incoming flows is equal to the summation of all outgoing flows, i.e. $\sum_{j} P_{j i, k}=\sum_{j} P_{i j, k}$, in other words, losses are negligible by considering highly efficient components (otherwise, losses must be modelled). Now, the power distribution's weight in (7) can be written as,

$$
\underset{P, x}{\operatorname{minimize}}\left[\left(\sum_{(i, j) \in \mathcal{A}} w_{i j} x_{i j}+\sum_{i \in \mathcal{N}} w_{i} x_{i}\right)+\left(\sum_{(i, j) \in \mathcal{A}}\left(w_{i j}^{\mathrm{kW}} \sum_{k \in K} P_{i j, k}\right)+\sum_{i \in \mathcal{N}}\left(w_{i}^{\mathrm{kW}} \sum_{k \in K} \sum_{j} P_{j i, k}\right)\right)\right]
$$


Similarly to (7), the first term in (8) is the summation of all fixed weights, and the second term is the summation of all variables weights (which depends on the amount of power flow converted/transferred). In (8), all power flows are expressed in terms of connections' power flows $P_{i j, k}$. The functional requirements are enforced with a set of connectivity constraints and the reliability requirements enforce sufficient number of components and connections to maintain the critical load $l$ of the distribution path $k$ (for which $y_{s l}=1$ ) connected to the generator $s$.

The first connectivity constraint is the flow balance (nodal equation) on every component $i \in \mathcal{N}$. Within the distribution path $k$, it will be assumed that only generator $s$ provides the power supply $d_{k}^{G E N}$ which is equal to the power demand needed on load $l$, or $d_{k}^{L O A D}$. Thus, for generators, $d_{k}^{G E N} \neq 0$ and $d_{k}^{L O A D}=0$, and for loads, $d_{k}^{G E N}=0$ and $d_{k}^{L O A D} \neq 0$. For the rest of components (step nodes), $d_{k}^{L O A D}=d_{k}^{G E N}=0$. Then, the flow balance for every component $i$ that is part of the distribution path $k$ can be written as,

$$
\sum_{i \mid(j, i) \in \mathcal{A}} P_{j i, k}-\sum_{i \mid(i, j) \in \mathcal{A}} P_{i j, k}+d_{k}^{L O A D}-d_{k}^{G E N}=0 \quad \forall i \in \mathcal{N}, \forall k \in K
$$

Constraint (9) is the nodal flow equation for every component $i \in \mathcal{N}$ that is part of distribution path $k \in K$. By convention, incoming flow and load demand is positive, while outgoing flow and generation power is negative. For every component $i$ that is selected, its incoming (and outgoing) connections and also be selected, then,

$$
x_{i j} \leq x_{i} \quad \forall(i, j) \in \mathcal{A}, \forall i \in \mathcal{N}
$$

Power flow is allowed only on selected connections and components (otherwise, it is 0 ). Given that distribution path $k$ delivers power demand $d_{k}^{L O A D}$ from a generator supplying $d_{k}^{G E N}$, the flow of the distribution path $k$ is limited to $d_{k}^{L O A D}$. For simplicity notation, let $d_{k}$ be equal to $d_{k}^{L O A D}$, then,

$$
\begin{array}{lr}
P_{i j, k} \leq d_{k} x_{i j} & \forall(i, j) \in \mathcal{A}, \forall k \in K \\
\sum_{j \in \mathcal{N}} P_{j i, k} \leq d_{k} x_{i} & \forall i \in \mathcal{N}, \forall k \in K
\end{array}
$$

Constraints (9)-(12) allow connectivity between power distribution components and connections. Now, the reliability constraints enforce that a sufficient number of components and connections are selected in order to ensure that the distribution path $k$ supplies its critical load from the corresponding generator. Recall that the template $\mathcal{G}=$ $\{\mathcal{N}, \mathcal{A}\}$ contains all permissible interconnections between components (from generators to loads) so that the critical load receives power from an adequate power conversion level or bus. A distribution path $k$ is, on its simplest structure, a series system that connects a generator with a critical load. Then, the reliability constraint of a distribution path $k$ (series system) can be written as,

$$
\left(\prod_{j \mid(i, j) \in k} x_{i j} r_{i j}\right)\left(\prod_{i \in k} x_{i} r_{i}\right) \geq r_{s l} \quad \forall k \in K
$$

where $r_{i}$ is the reliability of component $i, r_{i j}$ is the reliability of connection $i, j$, and $r_{s l}$ is the reliability target (equal to the reliability of the distribution path $k$ of the GS\&GLP step). In this case, (13) implements the distribution path $k$ as a series system. However, there are other ways to implement $k$. There are a number of network design approaches that can be used to build a power distribution system (network is a system of interconnected components in this context). These approaches are known as resilient designs [] and they propose a number of $w$ alternate paths in the case that any component or connection of the distribution path $k$ (series system) fails. A reliability constraint considering the alternate paths connecting the generator-load pair of the distribution path $k$ can be written as,

$$
1-\prod_{w}\left(1-\left(\prod_{j \mid(i, j) \in k} x_{i j} r_{i j}\right)\left(\prod_{i \in k} x_{i} r_{i}\right)\right) \geq r_{s l} \quad \forall k \in K
$$

The left-hand side of (14) is the reliability of a parallel-series system. Depending on the reliability target $r_{s l}$, (13) could not synthesize a distribution path and (14) will be introduced so that alternate paths are available and the reliability target $r_{s l}$ can be achieved. Up to this point, a minimum-weight topology for a power distribution system can be synthesized using (1)-(14). In order to get closer to a final implementation, additional constraints on the components' 
selection and location are included. These constraints allow to determine the impact of location and sizing on the overall system's construction.

Let the 2- dimensional space availability on a MEA airplane be represented with two straight lines, one (horizontal) spanning from the left- to the right-wing tips (wing line), and the other (vertical) crossing from the cockpit to the APU at the back of the plane (fuselage line). The components are located in any position along each one of these two lines. If selected, a component $i$ is located in a unique position $(m, n)$, then,

$$
\begin{array}{rlrl}
m_{i}^{\mathrm{MIN}} x_{i} & \leq m_{i} \leq m_{i}^{\mathrm{MAX}} x_{i} & & \forall i \in \mathcal{N} \\
n_{i}^{\mathrm{MIN}} x_{i} \leq n_{i} \leq n_{i}^{\mathrm{MAX}} x_{i} & & \forall i \in \mathcal{N}
\end{array}
$$

where $m_{i}, n_{i}$ are the abscissa and ordinate location variables for component $i ; m_{i}^{\mathrm{MIN}}, m_{i}^{\mathrm{MAX}}$ are the minimum and maximum abscissa location, and $n_{i}^{\mathrm{MIN}}, n_{i}^{\mathrm{MAX}}$ are the minimum and maximum ordinate location. If $x_{i}=0$ in (15)-(16) (component $i$ is not selected), the location variables $m_{i}, n_{i}$ are automatically set to 0 . The connection's length $l_{i j}$ connecting two components $i$ and $j$ (i.e. the length of connection $i, j$ ) can be determined by,

$$
l_{i j}=\left|m_{i}-m_{j}\right|+\left|n_{i}-n_{j}\right| \quad \forall(i, j) \in \mathcal{A}
$$

In (17), the length is measured as the sum of the absolute difference of the components' locations (1-Norm). There is no need to use Euclidean distance (2-Norm) because the components are only located along the two lines (the wing line, and the fuselage line). To ensure that each component has a unique location (no two components are placed on the same position), the constraint for unique location is,

$$
l_{i j} \geq \epsilon x_{i j} \quad \forall(i, j) \in \mathcal{A}
$$

where $\epsilon$ is a tolerance gap (distance gap) to force either $\left|m_{i}-m_{j}\right| \geq \epsilon$ or $\left|n_{i}-n_{j}\right| \geq \epsilon$ when connection $x_{i j}$ is selected (recall that components are located only along the wing line or along the fuselage line), hence $m_{i} \neq m_{j}$ or $n_{i} \neq n_{j}$. The selection of components is completed by including the location constraints (15)-(18). However, there is a set of constraints that are required to complete the selection of connections.

It is assumed that a component's location does not influence the component's size, i.e. component is selected only by the compliance of the connectivity and reliability constraints. In fact, adding other connectivity or reliability constraints would only intensify the search over the available components in the template $\mathcal{G}=\{\mathcal{N}, \mathcal{A}\}$. However, the connection's size is heavily influenced by the location of the components that it connects. There are two main characteristics that change in a connection depending on its length: 1) ampacity (gauge type), and 2) voltage drop. The selection of the connection's ampacity depends on the power flow transferred between any components $i, j$, while the voltage drop depends on the maximum permissible voltage drop and connection's length.

Consider a set $\mathcal{O}$ of standard wire gauges that are available for use. Let a Boolean $c_{i j, o}$ select a standard wire gauge $o(o \in \mathcal{O})$, then, each connection $x_{i j}$ that has been selected has a unique standard wire gauge, so,

$$
\sum_{o \in \mathcal{O}} c_{i j, o} \leq x_{i j} \quad \forall(i, j) \in \mathcal{A}
$$

Each standard wire gauge $o$ has an ampacity $I_{o}^{\mathrm{AWG}}$, a resistance per-unit-of-length $r_{o}^{\mathrm{AWG}}$, a weight per-unit-oflength $w_{o}^{\mathrm{AWG}} \cdot w_{o}^{\mathrm{AWG}}$ is different from $w_{i j}^{\mathrm{kW}}$ (inverse of the power density or power-to-weight ratio). Let the operating voltage of the connection $i, j$ be $V_{i j}^{\mathrm{OP}}$ (this voltage is pre-defined according to the power conversion components considered in the template $\mathcal{G}=\{\mathcal{N}, \mathcal{A}\}$ ). The ampacity constraint enforces that the current flowing through connection $i, j$ is less than or equal to the ampacity $c_{o}^{\mathrm{AWG}}$ of the wire gauge $o$, then,

$$
\frac{P_{i j}}{V_{i j}^{\mathrm{OP}}} \leq \sum_{o \in \mathcal{O}} I_{o}^{\mathrm{AWG}} c_{i j, o} \quad \forall(i, j) \in \mathcal{A}
$$

where $P_{i j} / V_{i j}^{\mathrm{OP}}$ represent the current flowing through connection $i, j$. Now, let the maximum permissible voltage drop (in p.u.) be $V_{p . u}^{\mathrm{DROP}}$. The voltage drop constraint ensures that the voltage drop across the connection is less than or equal to the maximum permissible voltage drop, then,

$$
\frac{P_{i j}}{V_{i j}^{\mathrm{OP}}} r_{o}^{\mathrm{AWG}} l_{i j} \leq V_{p . u .}^{\mathrm{DROP}} V_{i j}^{\mathrm{OP}}+V_{i j}^{\mathrm{OP}}\left(1-c_{o}^{\mathrm{AWG}}\right) \quad \forall(i, j) \in \mathcal{A}, \forall o \in \mathcal{O}
$$


where (21) is written using Big-M method. When $c_{o}^{\mathrm{AWG}}=1$ (i.e. a wire gauge is chosen for a selected connection), constraint (21) enforces the actual voltage drop to be less than or equal to $V_{p . u .}^{\mathrm{DROP}} V_{i j}^{\mathrm{OP}}$; if $c_{o}^{\mathrm{AWG}}=0$, the actual voltage drop is arbitrarily set. In summary, constraints (15)-(21) are introduced as location and selection constraints and these constraints aim to determine the cabling size of the power distribution system. If these constraints were not considered in the design, the topology's weight would still need to be adjusted in further refinement steps during the PBD. In the following section, the linearization of constraints (6), (13), (14), and (21) will be discussed.

\section{Linear Transformations on Non-linear constraints}

The reliability constraints in (6), (13), and (14) are non-linear because of the products between Boolean variables. In fact, these Boolean allow reliabilities to be set to 0 when the components or connections are not selected. In the case of (6), the products can be linearized by taking the logarithm on both sides of the constraint, then,

$$
\sum_{s} y_{s l} \ln \left(1-r_{s l} r_{s}\right) \leq \ln \left(1-r_{\text {TARGET }, l}\right) \quad \forall l
$$

where the Boolean $y_{s l}$ has been taken outside the logarithm because $\ln \left(1-y_{s l} r_{s l} r_{s}\right)=0$ when $y_{s l}=0$, then if $y_{s l}$ is multiplied by $\ln \left(1-r_{s l} r_{s}\right)$ the result is the same for $y_{s l}=0$ (same results are obtained for $y_{s l}=1$ ). Similarly, the products in (13) can be linearized by taking the logarithm on both sides of the constraint, then,

$$
\sum_{j \mid(i, j) \in \mathcal{A}} z_{i j, k} \ln \left(r_{i j}\right)+\sum_{i \in \mathcal{N}} z_{i, k} \ln \left(r_{i}\right) \geq \ln \left(r_{s l}\right) \quad \forall k \in K
$$

where $z_{i j, k}$ and $z_{i, k}$ are Boolean variables that determine if a connection $x_{i j}$ and component $x_{i}$ are used for a distribution path $k$ (note that constraint (13) is a product series over connections $(i, j) \in k$ and components $i \in k$ ). Linearization of constraint (14) is more complicated (reliability of a parallel-series system). Let a Boolean $q_{w}$ be set $\left(q_{w}=1\right)$ if an alternate path $w$ is constructed. Also, let the series system of the inner product have a reliability $r_{q}$ and let this series system be linearized in the same way as presented in the left-hand side of (23). Then, the linearization of a parallel-series system is,

$$
\begin{array}{ll}
\sum_{w} q_{w} \ln \left(1-r_{q}\right) \leq \ln \left(1-r_{s l}\right) & \forall k \in K \\
\sum_{j \mid(i, j) \in \mathcal{A}} z_{i j, k} \ln \left(r_{i j}\right)+\sum_{i \in \mathcal{N}} z_{i, k} \ln \left(r_{i}\right)=\ln \left(r_{q}\right) & \forall k \in K
\end{array}
$$

Finally, the left hand-side of (21) presents a product of two continuous variables, $P_{i j}$ (that depends on the amount of power flow) and $l_{i j}$ (that depends on the location of components). There are a number of alternatives for linearizing products of continuous variables (this product can be thought of as a surface). One alternative is to discretize the product so that a small section is represented by a point in the middle of the section. Other alternative is to use surface approximations [17] in order to have intermediate values. A third alternative is to use McCormick envelopes [18] which are linear envelopes that provides lower and upper boundaries for each section. In any case, the product $P_{i j} l_{i j}$ is replaced by a third variable which is solved with the linearization method that fits best.

\section{Case Study}

A case study is presented to exemplify the use of the design formulation detailed in Section III. A MEA power distribution system is synthesized to fulfill the requirement of supplying electrical power to 4 loads (total demand is $125 \mathrm{~kW}$ ) in a small MEA. The loads' requirements (power demands and reliabilities) are shown in Table 1.

Table 1 Load requirements

\begin{tabular}{clcccc}
\hline \hline \multirow{3}{*}{ Loads } & $L_{1}$ & $L_{2}$ & $L_{3}$ & $L_{4}$ \\
\cline { 3 - 6 } & $L_{l}[\mathrm{~kW}]$ & 50 & 25 & 40 & 10 \\
& $1-r_{\mathrm{TARGET}, l}$ & $1.0 \times 10^{-9}$ & $1.0 \times 10^{-6}$ & $1.0 \times 10^{-6}$ & $1.0 \times 10^{-6}$ \\
\hline \hline
\end{tabular}


The load's reliability target is expressed as the probability of not being supplied with electrical power, i.e. $1-r_{\text {TARGET, } l}$. Loads $L_{1}$ to $L_{3}$ are LV DC, and $L_{4}$ is the only HV DC load. The templates $\mathcal{G}=\{g, \mathcal{E}\}$ for the GS\&GLP and $\mathcal{G}=\{\mathcal{N}, \mathcal{A}\}$ for the PDD steps are shown in Fig. 1. Because there are LV DC and HV DC loads, the power distribution system is assumed to have two voltage levels (high voltage DC and low voltage DC) and one power conversion level to convert HV DC to LV DC. Power conversion is performed by HV/LV DC power converters which can be fed from several generators (power sources) and its LV DC output can be paralleled to other power converters to supply the same load bus. In the LV DC side, LV DC buses distribute the power to the LV DC loads. In the HV DC side, there are source matrix contactors (HV box) that distribute the generation power. The source matrix contactor (HV box) is a device that receives power inputs from multiple generators, routes the received power to the HV/LV DC power converters, and supplies power to the HV DC loads directly. It is assumed that the source matrix contactor (HV box) is configured in such a way that there is no risk of cross-connecting generators. Thus, there are three types of power distribution components: source matrix contactor (HV box), HV/LV DC power converter, and LV bus.
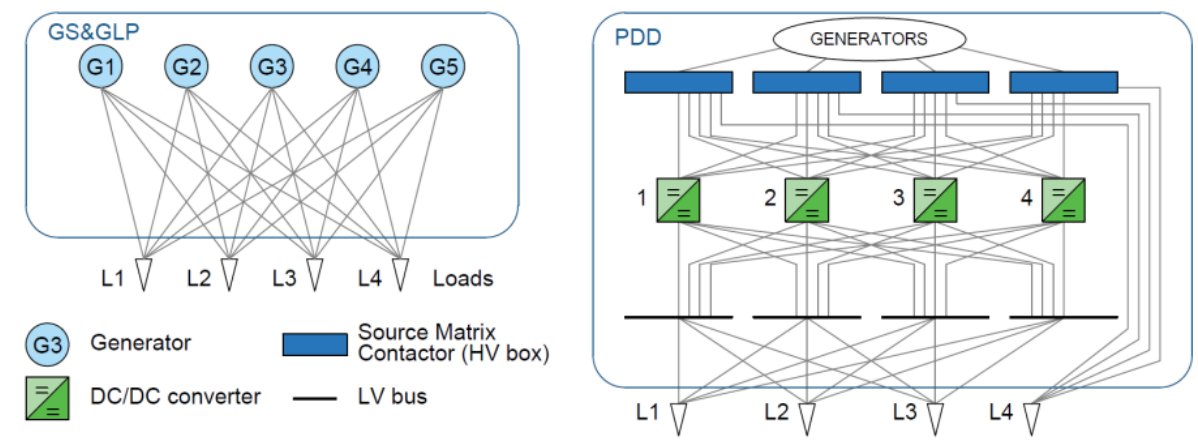

Fig. 1 Templates for GS\&GLP step (left), and PDD step (right)

The parameters of the generators are listed in Table 2 (all generators have the same range of parameters). The generator's power rating $P_{S}^{G}$ spans from $25 \mathrm{~kW}$ to $150 \mathrm{~kW}$. The generator's weight $w_{s}$ is modelled as a function of the power rating $P_{s}^{G}$, i.e. $w_{s}\left(P_{s}^{G}\right)$, and this function is linear. The generator's reliability $r_{s}$ is presented as the probability (of generator $s$ ) of not supplying power, i.e. $1-r_{s}$. The selected generators can have different power ratings and weights, depending on the optimal solution found in the GS\&GLP formulation. The reliability of the distribution path $r_{s l}$ is assumed to be in the range of 0.9000 to 0.9997 (it can also be expressed as $1-r_{s l}$, or the probability of the distribution path of not delivering power from generator $s$ to load $l$. The GS\&GLP optimization problem is:

$$
\begin{aligned}
& \text { min Eq. (1) } \\
& \text { subject to Eqs. (2) - (5), Eq. (22) }
\end{aligned}
$$

Table 2 GS\&GLP input specification for MEA design

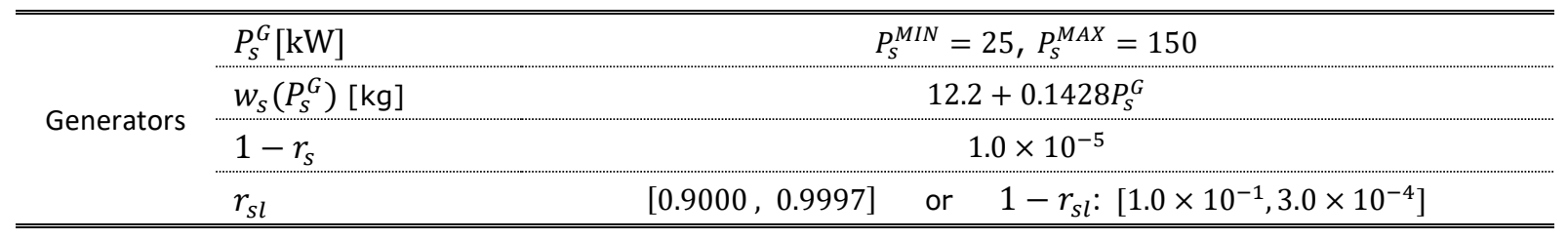

The parameters of the power distribution components and connections are listed in Table 3 (all components of the same type have the same parameters). The fixed weight for components and connections $w_{i}, w_{i j}$ is in $\mathrm{kg}$, and the variable weight $w_{i}^{\mathrm{kW}}, w_{i j}^{\mathrm{kW}}$ is in $\mathrm{kg} / \mathrm{kW}$. The reliabilities $r_{i}$ and $r_{i j}$ are expressed as the probability of not performing the intended function (or the probability of failure) $1-r_{i}$ and $1-r_{i j}$. The PDD optimization problem is:

min Eq. (8)

subject to Eqs. (9) - (12), Eq. (23) or (24) - (25), Eqs. (15) - (21) 
Table 3 PDD input specification for MEA design

\begin{tabular}{cccccc}
\hline \hline & & HV box & Converters & LV DC buses & Connections $(i j)$ \\
Power & $w_{i}, w_{i j}[\mathrm{~kg}]$ & 1.8 & 8.5 & 1.8 & 0.2 \\
distribution & $w_{i}^{\mathrm{kW}}, w_{i j}^{\mathrm{kW}}[\mathrm{kg} / \mathrm{kW}]$ & 0.1 & 0.3 & 0.1 & 0.05 \\
\cline { 2 - 3 } & $1-r_{i}, 1-r_{i j}$ & $5.0 \times 10^{-6}$ & $1.0 \times 10^{-4}$ & $5.0 \times 10^{-6}$ & $2.0 \times 10^{-6}$ \\
\hline \hline
\end{tabular}

The position (location) of the generators and loads is fixed. The four loads are located according to the following arrangement: two LV DC loads are located in the wings (one on the left wing, and the other on the right wing), one LV DC load is located in the airplane's cockpit, and the HV DC load is located at the back. The generators can be located in the engine area (left and right), and the location in the tail is also available. The rest of the power distribution components are located according to the PDD formulation. The MEA power system architecture synthesis is solved in two steps: GS\&GLP problem (26)-(27), and PDD problem (28)-(29). The case study is solved using a Windows High Spec PC Intel Xeon 64-bit 3.60GHz running CPLEX Studio IDE 12.9.0 [19]. The results of the MEA synthesis is shown in Fig. 2.

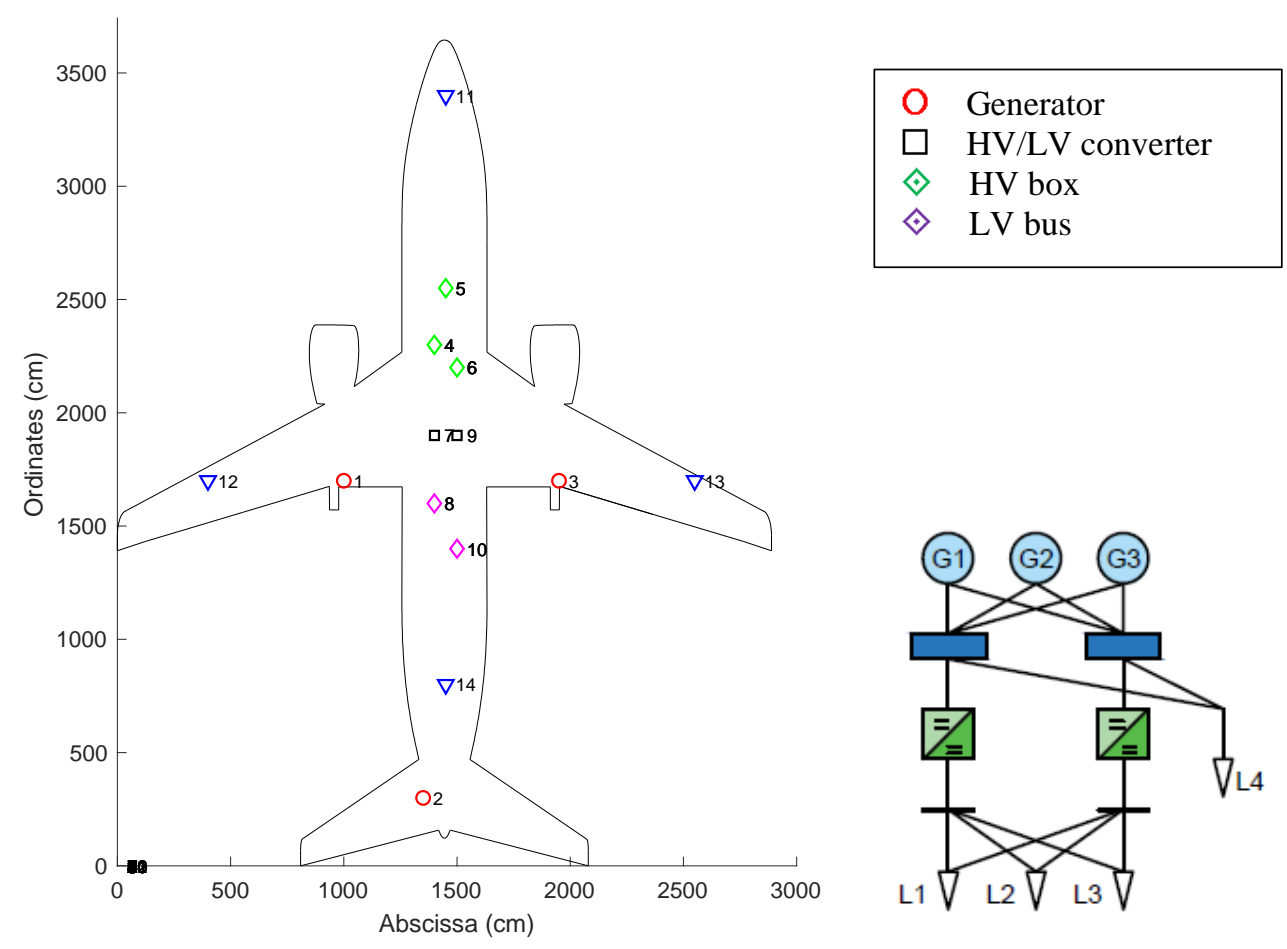

Fig. 2 Results for MEA power system architecture synthesis, location (left), topology (right)

The topology of the MEA power system is shown in Fig. 2. The connections shown in Fig. 2 only reflects the topology's connectivity and the optimal location for the power distribution components (for illustrative purposes). In reality, the connections are wired only along the wing-line and the fuselage-line. The optimal solution has taken this condition into consideration and the optimal solution has been reached.

\section{Conclusion}

An optimization-based formulation for the design and synthesis of a MEA power system architecture that has minimum weight has been proposed. Functional constraints have been translated to a set of connectivity constraints and the safety requirements have been translated to a set of reliability constraints. Given the non-linearity of the reliability constraints which ensures that critical loads are supplied at all times, linearization is performed to allow mixed integer linear programming solvers to find an optimum solution. A set of location and selection constraints are introduced to improve the accuracy of the minimum weight solution. A case study for a small MEA plane has been presented. 


\section{Acknowledgments}

This work is funded by the INNOVATIVE doctoral programme. The INNOVATIVE programme is partially funded by the Marie-Curie Initial Training Networks (ITN) action (project number 665468), and partially by the Institute for Aerospace Technology (IAT) at the University of Nottingham.

\section{References}

[1] G. Buticchi, S. Bozhko, M. Liserre, P. Wheeler, and K. Al-Haddad, "On-board microgrids for the more electric aircraft Technology review,” IEEE Trans. Ind. Electron., vol. 66, no. 7, pp. 5588-5599, 2019.

[2] B. Karanayil, M. Ciobotaru, and V. Agelidis, "Power Flow Management of Isolated Multiport Converter for More Electric Aircraft," IEEE Trans. Power Electron., vol. 8993, no. c, pp. 1-1, 2016.

[3] I. Christou, A. Nelms, I. Cotton, and M. Husband, "Choice of optimal voltage for more electric aircraft wiring systems," IET Electr. Syst. Transp., vol. 1, no. 1, pp. 24-30, 2011.

[4] X. Roboam, B. Sareni, and A. De Andrade, "More Electricity in the Air," Industrial Electronics Magazine, IEEE, vol. 6, no. 4, pp. 6-17, Dec-2012.

[5] A. Tardy et al., "Towards More Optimization for Aircraft Energy Conversion Systems," in MEA 2015 - More Electric Aircraft-, 2015, no. February, p. 16.

[6] J. Chen, C. Wang, and J. Chen, "Investigation on the selection of electric power system architecture for future more electric aircraft," IEEE Trans. Transp. Electrif., vol. 4, no. 2, pp. 563-576, 2018.

[7] J. Brombach, A. Lucken, B. Nya, M. Johannsen, and D. Schulz, "Comparison of Different Electrical HVDC- Architectures for Aircraft Application," in Electrical Systems for Aircraft, Railway and Ship Propulsion (ESARS), 2012, 2012, pp. 1-6.

[8] L. Jun and X. Huibin, "Reliability Analysis of Aircraft Equipment Based on FMECA Method," in 2012 International Conference on Solid State Devices and Materials Science, 2012, vol. 25, pp. 1816-1822.

[9] R. D. Telford, S. J. Galloway, and G. M. Burt, "Evaluating the reliability \& availability of more-electric aircraft power systems," in Proceedings of the Universities Power Engineering Conference, 2012.

[10] H. Ounis, B. Sareni, X. Roboam, and A. De Andrade, "Multi-level integrated optimal design for power systems of more electric aircraft," in Mathematics and Computers in Simulation, 2016, vol. 130, pp. 223-235.

[11] P. Nuzzo et al., "A contract-based methodology for aircraft electric power system design," IEEE Access, vol. 2, pp. 1-25, 2014.

[12] J. Finn, P. Nuzzo, and A. Sangiovanni-Vincentelli, “A mixed discrete-continuous optimization scheme for Cyber-Physical System architecture exploration," in 2015 IEEE/ACM International Conference on Computer-Aided Design (ICCAD), 2015, pp. 216-223.

[13] A. Pinto, S. Becz, and H. Reeve, "Correct-by-Construction Design of Aircraft Electric Power Systems," in 10th AIAA Aviation Technology, Integration, and Operations (ATIO) Conference, 2010, pp. 1-11.

[14] A. Sangiovanni-Vincentelli, "Quo vadis, SLD? Reasoning about the trends and challenges of system level design," Proc. IEEE, vol. 95, no. 3, pp. 467-506, 2007.

[15] S. Becz, A. Pinto, L. E. Zeidner, R. Khire, A. Banaszuk, and H. M. Reeve, "Design system for managing complexity in aerospace systems," in 10th AIAA ATIO/ISSMO Conference, 2010, pp. 1-7.

[16] P. Nuzzo, J. Finn, M. Mozumdar, and A. Sangiovanni-vincentelli, "Platform-Based Design Methodology and Modeling for Aircraft Electric Power Systems," in Proceedings of Green Energy and Systems Conference, 2013, pp. 1-7.

[17] C. D'Ambrosio, A. Lodi, and S. Martello, "Piecewise linear approximation of functions of two variables in MILP models," Oper. Res. Lett., vol. 38, no. 1, pp. 39-46, Jan. 2010.

[18] M. L. Bergamini, P. Aguirre, and I. Grossmann, "Logic-based outer approximation for globally optimal synthesis of process networks," Comput. Chem. Eng., vol. 29, no. 9, pp. 1914-1933, Aug. 2005.

[19] “IBM ILOG CPLEX Optimization Studio.” [Online]. Available: https://www.ibm.com/products/ilog-cplex-optimizationstudio. 